\title{
Paris als non-lieu: Migration im Werk von Marina Zwetajewa, 1925-1932
}

Als Marina Zwetajewa, die zusammen mit Anna Achmatowa zu den größten russischen Dichterinnen des zwanzigsten Jahrhunderts zählt, im Sommer 1909 zum ersten Mal nach Paris kam, war sie sechs Jahre alt. Nach dem frühen Tod ihrer Mutter, der begabten Pianistin Maria Meyn, folgten für die nur vierzehnjährige Marina und ihre jüngere Schwester Anastasia mehrere jeweils monatelange Studienaufenthalte in Deutschland und Frankreich. Marina, die dreisprachig aufwuchs und neben Russisch auch Deutsch und Französisch beherrschte, kam in die französischen Hauptstadt mit dem Ziel, Vorlesungen zur altfranzösischen Literatur an der Sorbonne zu hören. Diese wenigen Monate sollten für Zwetajewa die einzigen sein, in denen sie in Paris wohnte. In dieser Zeit entstand ein kurzes Gedicht über Paris, das sehr lange der einzige Text blieb, den sie über diese Stadt schrieb. Im Gegensatz dazu stehen mehrere Gedichte, die sie später den anderen Stationen ihrer Emigration - Prag und Berlin - widmen sollte. Bereits in diesem frühen Gedicht drückte sie ihre Zurückhaltung gegenüber Paris aus. Es ist sicherlich noch kein reifes Gedicht, doch bereits hier reflektiert die junge Dichterin den illusorischen Status der Stadt als Sehnsuchtsort, den sie gleich zu Beginn postuliert: «im großen und fröhlichen Paris / die gleiche heimliche Wehmut.» ${ }^{1}$ Mit der ersehnten Ankunft in der Metropole endet die auf sie bezogene Nostalgie nicht, im Gegenteil, sie wird durch die Begegnung mit Paris noch verstärkt. Nicht die schönen Boulevards oder die Verliebten, die im Gedicht vorkommen, sind die Bezugspunkte für das lyrische Ich, sondern ein zunächst unsichtbarer kultureller Text von Paris. In zwei Strophen erwähnt Zwetajewa das Werk von Edmond Rostand und die weltberühmte Schauspielerin Sarah Bernhardt, die sie noch aus Kindertagen kannte und verehrte. Dennoch geht Zwetajewa in ihrer ambivalenten Beschreibung der Stadt als Sehnsuchtsort, der zugleich ein Ort der Einsamkeit ist, über die Konventionen des früheren Modernismus hinaus, da sie Paris mit anderen Metropolen in Verbindung bringt, und zwar mit dem «verlassenen» Moskau. Moskau wird von ihr als Gegengewicht zum Pariser urbanen Raum dargestellt: «In dem großen und freudigen Paris träume ich von Gras und Wolken.» ${ }^{2}$ Wie der

1 Marina Zwetaejewa: Sochinenija [Gesammelte Schriften]. Bd. I der Gesammelten Werke. Moskau: Chudozestvennaja Literatura 1990, S. 77. Meine Übersetzung.

2 Marina Zwetajewa: Sochinenija, S. 78. Meine Übersetzung.

Ә Open Access. (C) 2020 Natasha Gordinsky, publiziert von De Gruyter. (c) BY-NC-ND Dieses Werk ist lizenziert unter der Creative Commons Attribution-NonCommercial-NoDerivatives 4.0 Lizenz.

https://doi.org/10.1515/9783110679366-002 
folgende Beitrag zeigen möchte, blieb Moskau auch sechzehn Jahre später, als Zwetajewa mit ihrer Familie nach Paris übersiedelte, für sie ein Gegengewicht zu Paris, trotz der Tatsache, dass sie sich in der sowjetischen Hauptstadt für sich selbst aus politischen Gründen keine Zukunft vorstellen konnte. Ich möchte sowohl einer poetischen als auch einer historischen Frage nachgehen, die meines Erachtens in der Forschung zum Werk Zwetajewas noch nicht gestellt wurde, nämlich der Frage, aus welchen Gründen die Dichterin fast vollständig auf ein Schreiben über Paris verzichtet hat, obwohl sie fast fünfzehn Jahre ihres Lebens in Frankreich verbrachte. Auch wenn sie in Paris selbst nur wenige Monate lebte und auch da nur am Rande der Stadt, wurde die Stadt an der Seine, so meine These, für Zwetajewa zum Exilort par excellence, oder in einer Paraphrase ihrer eigenen Gedanken, zum Ort der Nicht-Emigration. ${ }^{3}$ Warum aber beziehen sich nur wenige von ihren literarischen Texten, die zwischen 1925 und 1939 verfasst wurden, explizit auf Frankreich oder setzen sich mit der französischen Kultur auseinander? Gerade diese Texte, die zu diversen Gattungen gehören, nämlich Lyrik, essayistische Prosa und Tagebücher, enthalten ihre wichtigsten Überlegungen zur Bedeutung der Emigration. Der erste Teil dieses Aufsatzes befasst sich mit dem ersten langen Gedicht, das Zwetajewa über Paris verfasste, «Das Treppengedicht.» Der zweite Teil untersucht eine poetische und intellektuelle Begegnung, die 1928 in Paris zwischen zwei Hauptfiguren des russischen Modernismus Marina Zwetajewa und Natalia Gontscharowa - stattfand. Diese Begegnung wird in Zwetajewas langem Essay «Natalja Gontscharowa (Leben und Werk)» dokumentiert. Der letzte Teil dieses Beitrags reflektiert schließlich Zwetajewas kulturelle Ambivalenz zu Frankreich im Kontext ihrer Emigrationserfahrung.

\section{In einem Treppenhaus am Stadtrand von Paris}

Zur Zeit ihrer Übersiedlung nach Paris wurde Zwetajewa bereits als eine der führenden Stimmen der russischen Dichtung anerkannt, und zwar sowohl in der Emigration als auch in der Sowjetunion, wo es vor allem Boris Pasternak war, der ihren zweiten, 1922 in Berlin publizierten Gedichtband entdeckte und öffentlich pries. ${ }^{4}$ Nach höchst produktiven Jahren, die Zwetajewa in der tschechischen

3 Die Slawistin Ute Stock untersucht in ihrem Buch die wesentlichen Gründe dafür, warum in der Sekundärliteratur über Zwetajewa die Frage des Exils randständig geblieben ist. Siehe dazu: Ute Stock: The Ethics of the Poet: Marina Tsveateva's Art in the Light of Conscience. Leeds: Maney Publishing 2005, S. 59-62.

$4 \mathrm{Zu}$ den unterschiedlichen Stationen von Zwetajewas Emigration siehe: Marie-Louise Botte: Marina Zwetajewa in der Emigration 1922-1939. In: Simon-Pierre Hamelin (Hg.): 101 rue Condorcet, 
Emigration verbrachte, wo sie von der Regierung mit einem Stipendium gefördert wurde, verließ sie 1925 ihre Lieblingsstadt Prag. Sie fand mit ihren Kindern Ariadna, die später Kunstunterricht von Natalja Gontscharowa erhielt, und dem kleinen Georgiy Aufnahme in der Rue Rouve in der Wohnung ihrer Freundin Olga Chernowa, die sich mit Zwetajewas Familie bereits in Prag angefreundet hatte und die es später durch ihre Kontakte in Paris schaffte, dieser ein Visum zu besorgen. Wie viele russische Emigranten wohnte auch Olga Chernowa am Stadtrand von Paris, in einem armen Arbeiterviertel. Von den drei Zimmern ihrer kleinen Wohnung bot sie eines Zwetajewa und ihren Kindern an. Nachdem Zwetajewa einige Jahre in der schönsten Umgebung von Prager Vororten gelebt hatte, waren ihre ersten Eindrücke von dem Arbeiterviertel nun bedrückend, wie sie ihrer langjährigen tschechischen Freundin Anna Teskowa berichtete: «Das Viertel, in dem wir wohnen ist furchtbar [...] Verrotteter Kanal, den Himmel kann man wegen der Industrieanlagen nicht sehen, überall Ruß und Lärm (wegen der Lastkraftwagen). Spazieren kann man nirgendwo - kein einziger Busch hier.» ${ }^{5}$ Die engen Lebensverhältnisse waren für Zwetajewa eigentlich nichts Neues, denn seit der Oktoberrevolution lebte sie mit ihrer Familie fast ununterbrochen in großer Armut. Aber dieser Grad ihrer eigenen Armut und das enge Beisammensein mit den anderen armen Arbeiterfamilien in dem Viertel bedeutete für sie eine neue und ergreifende Erfahrung. Auch der Kontrast zwischen dem Paris ihrer Jugend und dem Paris der Emigrationszeit war für sie zu groß, um davon nicht innerlich betroffen zu sein.

Bereits in diesen Monaten beschloss sie, diese existenzielle Erfahrung in poetischer Form zu reflektieren und sie begann, an einem langen Gedicht mit dem Titel «Wie das Treppenhaus lebt und arbeitet» zu schreiben. Die Vollendung der Arbeit an diesem Text wurde aus überraschenden und erfreulichen Gründen verzögert, denn das Honorar von einem in Berlin publizierten Gedichtband erlaubte es ihr, für sich und ihre Familie sechs Monate lang eine Wohnung an der Atlantikküste, in dem kleinen Ort St. Gilles-Croix-de-Vie, zu mieten. Im Sommer 1926 entwickelte sich an eben diesem Ort der zutiefst poetische und intime Briefwechsel, den Marina Zwetajewa und Rainer Maria Rilke in deutscher Sprache führten; eine Begegnung, die dank der großzügigen Vermittlung von Boris Pasternak stattgefunden hatte und Rilke veranlasste, noch kurz vor seinem Tod Zweta-

Clamar. Übersetzung von Regina Keil-Sagawe. Hamburg: Osburg Verlag 2017, S. 93-115, hier S. 104.

5 Marina Zwetajewa: Briefwechsel mit Anna Teskowa. Brief vom 7. Dezember 1925. Der Nachlass ist digitalisiert und einsehbar unter: http://rulibrary.ru/tsvetaeva/pisma_chast_1/194 [8.08.2019]. Meine Übersetzung. 
jewa als eine ihm ebenbürtige Dichterin anzuerkennen. ${ }^{6}$ Zwetajewas Erfahrungen in Frankreich sind nicht ohne ihre sowohl in biographischer als auch in poetischer Hinsicht höchst bedeutende Beziehung zu Rilke zu verstehen, auch wenn diese für sich genommen nicht Gegenstand der folgenden Überlegungen sein kann. Für Zwetajewa verkörperte Rilke nicht nur die deutschsprachige Dichtung, sondern die Dichtung insgesamt, wie sie in den Briefen an ihn mehrmals betonte. Rilkes Anerkennung, die auch in seiner an die russische Dichterin adressierten Elegie - eines seiner letzten Gedichte überhaupt - in kristalliner Form zum Ausdruck kam, war für Zwetajewa ein weiterer Beweis, dass Rilke in ihr eine Gleichgesinnte gesehen hat. Beide schufen ihr dichterisches Werk in einer Sprache, die jenseits der Grenzen nationaler Literaturen existierte. Zugleich bedeutete dieser Briefwechsel für Zwetajewa eine poetische Auserwählung der Art, die für sie in der französischen literarischen Szene der 1920er Jahre undenkbar war. Es ist $\mathrm{zu}$ vermuten, dass die so einzigartige Verbindung mit Rilke Zwetajewas Wahrnehmung ihrer eigenen kulturellen Isolation in Frankreich noch verstärkt hat. ${ }^{7}$ Tatsächlich blieb Zwetajewa für das französische literarische Milieu auch zehn Jahre nach diesem Briefwechsel noch völlig unbekannt. In dem geistigen Raum zwischen Isolation und Anerkennung vollendete sie im Sommer 1926 - dem wahrscheinlich glücklichsten all ihrer Jahre in Frankreich überhaupt - ihr oben erwähntes «Treppengedicht».

Es waren dies, wie erwähnt, die einzigen Verse über Paris, die in den fast fünfzehn Jahren von Zwetajewas Exil in Frankreich entstanden. Liest man das Gedicht heute ohne jede Kenntnis des lebensgeschichtlichen Kontextes, in dem es entstand, kann man Paris als Sehnsuchtsort ihrer Jugend überhaupt nicht erkennen, zumal kein bekanntes topographisches Merkmal der Metropole an der Seine auftaucht. Aus Zwetajewas Sicht war dies kein Zufall - denn was in ihrem Text dargestellt wird, ist mit Absicht alles andere als glanzvoll, hier geht es allein um die Kehrseite von Paris. Der Protagonist der Verse ist kein Mensch, sondern ein Treppenhaus, in dem sich das ganze Leben der Einwohner der Pariser Vorstädte abspielt. Dieser Raum flüchtiger Alltags- und Zufallsbegegnung ist im Gedicht der Zeuge von heimlichen erotischen Begegnungen, von «Zwängen des Alltags», wie es im Gedicht heißt, und immer wieder von der Armut der Nachbarn, die alle aus ganz unterschiedlichen Ländern nach Paris kamen. Das Alltagsleben der Einwohner wird auf metonymische Weise durch den Lärm und die Geräusche auf den Treppen dargestellt. So komponiert die Dichterin eine ganze Partitur dieser

6 Siehe dazu: Konstantin Asadowski: Orpheus und Psyche. Vorwort. In: Rainer Maria Rilke und Marina Zwetajewa: Ein Gespräch in Briefen. Frankfurt a. M.: Suhrkamp 1998, S. 7-45, hier S. 7-41. 7 Einen so intensiven Briefwechsel, Umgang und Austausch mit ihren Zeitgenossinnen und Zeitgenossen in Frankreich gab es nicht. 
Treppenmusik: «Was - die Etage? Mit Husten: / Direkt im Bund. / Auch unsre Stufen / Tun Tiefen kund. / Husten - Krampf und Tränen, / Kichern, stöhnen, / Keuchen, röcheln. Schwarze Treppe / Hat auch Höhen.» ${ }^{8}$ Entscheidend ist auch die Darstellung von Gerüchen, weil das Essen, oder genauer gesagt, der Mangel an Speisen eine große Rolle im Gedicht spielt: «Teuer ist alles! / Hungrig? Mußt raffen! / Schlafen? Nein, laß es - / Essen beschaffen!». ${ }^{9}$ Im ersten Teil des Gedichts sind die Treppen als die realen schwarzen Treppenstufen im Haus der Armut zu verstehen. Im zweiten Teil der Dichtung entfalten sie jedoch auch eine metaphysische Bedeutung, indem sie auf die biblischen Treppen in Jakobs Traum verweisen: «Ach, der Jakobstraum! Gute alte Zeit!» ${ }^{10}$ Laut der biblischen Erzählung träumte Jakob während seiner Flucht aus Beer-Sheva von der Himmelsleiter: «Und ihm träumte: Da, eine Leiter gestellt auf die Erde, ihr Haupt an den Himmel rührend, und da, Boten Gottes steigen auf, schreiten nieder an ihr.» ${ }^{11}$ Auch wenn diese Engel in Zwetajewas Haus im Arbeiterviertel keinen Platz finden, deutet das lyrische Subjekt die Existenz der metaphysischen Sphäre an. Aber im Gegensatz zum biblischen Text wird das Haus in der banlieue nicht ins «Haus des Gottes» verwandelt. Das Gedichtet bietet keine Erlösung für die Einwohner; zugleich fungiert aber die Jakobsleiter als Brücke zu einer idealen Welt. Diese wird im zweiten Teil als die Abschaffung der dinglichen Welt verstanden.

Hinter dem narrativen Kern des Gedichts verbirgt sich nämlich auch eine philosophisch-politische Dimension, die sich auf die Abschaffung der materiellen Sachen durch eine magische Verwandlung der alltäglichen Dinge in ihre Rohstoffe bezieht. Da die Menschen keinen Weg aus der Armut herausfinden können, imaginiert Zwetajewa die Situation einer umgekehrten Rebellion - die Dinge übernehmen eine aktive Rolle und rebellieren gegen die Menschen, die sie ausbeuten. Der Versuch der Dinge, in einen oder vielmehr in ihren ursprünglichen Zustand zurückzukehren, ist der wichtigste philosophische Aspekt, den Zwetajewa in diesem Text zum Ausdruck bringt. Erst mit der anbrechenden Nacht beruhigt sich das ganze Haus und mit ihm auch der Alltagslärm. Plötzlich werden die Dinge, die den Menschen im Alltag dienen, wach und streben danach, sich von ihrer eigenen «Unbeweglichkeit» zu befreien. Dabei geht es Zwetajewa aber nicht um eine bloße Anthropomorphisierung der Dinge, sondern recht eigentlich um das Gegenteil, nämlich um das Enteignen der menschlich anmutenden Seiten von allen gewöhnlichen Sachen. Doch gerade diese Intention der Dichterin wird

8 Marina Zwetajewa: Gruß vom Meer. Übersetzung von Felix Phillip Ingold. München: Hanser Verlag 1994, S. 88.

9 Ebda., S. 86.

10 Ebda., S. 89.

11 Bibel, Gen 28. 13, Buber-Rosenzweig Übersetzung, 1929. 
im Gedicht paradoxerweise durch die anthropomorphische Metaphorik ermöglich: «Hof möchte Vorstadt werden / Mit Blumen und mit Beeren.» ${ }^{12}$ Die verborgene «Würde der Dinge» kann nur dann zurückgewonnen werden, so besagt das Gedicht, wenn die Dinge in ihr ursprüngliches Material zurückgekehrt sind. Dies bedeutet aber in diesem Fall, dass die Dinge ihr «Wesen als Warenstruktur» ${ }^{13}$ verlieren, da sie sich zur Natur, also in den Rohstoff ihrer Entstehung verwandeln: «Sagt das Vitrinen Glas: / - Bin Sa-and! Bin Quarz!» ${ }^{14}$ An dieser Stelle ist festzustellen, in welch frappierender Weise Zwetajewas lyrisch-philosophischer Ansatz ein marxistisches Argument entfaltet. Nicht zufällig wird der Name von Karl Marx in ihrem Gedicht gleich zu Beginn erwähnt, denn Zwetajewa geht es auch um eine poetische Deutung dessen, was im Marxismus «Verdinglichung» genannt wird. Nur drei Jahre zuvor widmete der ungarisch-jüdische Denker Georg Lukács dem Begriff der Verdinglichung in seinem 1923 erschienenen Buch Geschichte und Klassenbewusstsein ein ganzes Kapitel. Seine Erweiterung des marxschen Denken, das auf die Objektivierung der Verhältnisse der arbeitenden Menschen durch die produzierten Waren verweist, erleuchtet Zwetajewas philosophisches Argument:

\footnotetext{
Diese rationelle Objektivierung verdeckt vor allem - den qualitativen und materiellen unmittelbaren Dingcharakter aller Dinge. Indem die Gebrauchswerte ausnahmslos als Waren erscheinen, erhalten sie eine neue Objektivität, eine neue Dinghaftigkeit, die sie zur Zeit des bloßen gelegentlichen Tausches nicht gehabt haben, in der ihre ursprüngliche, eigentliche Dinghaftigkeit vernichtet wird, verschwindet sagt Marx, «entfremdet nicht nur die Individualität der Menschen, sondern auch die der Dinge [...]». ${ }^{15}$
}

Der Aufstand der Dinge in Zwetajewas «Treppengedicht» ist also als Rebellion gegen die Vernichtung ihrer eigentlichen «Dinghaftigkeit» zu verstehen. Und so liest sich das Streben von Dingen zurück in das Material, aus dem sie produziert wurden, als Zwetajewas poetischer Versuch, die Individualität der Dinge wiederzugewinnen: «Was die Dinge, klug geworden lernten, / Ist - sie müssen Bruch mit Bruch vergelten.» ${ }^{16}$ Demzufolge wäre auch die metonymische Darstellung der Mitbewohner des Hauses durch die von ihnen produzierten Waren als eine Manifestierung der Objektivierung der Menschen zu lesen. Der Vorwurf der Dinge gegen die Menschen - «Ihr - mit Gegenständen und Begriffen» - fungiert auch

12 Marina Zwetajewa: Gruß vom Meer, S. 92.

13 Georg Lukács: Geschichte und Klassenbewusstsein. Studien über marxistische Dialektik. Berlin: Malik-Verlag 1923, S. 97.

14 Marina Zwetajewa: Gruß vom Meer, S. 92.

15 Georg Lukács: Geschichte und Klassenbewusstsein, S. 104.

16 Marina Zwetajewa: Gruß vom Meer, S. 94. 
als eine Kritik der kapitalistischen Gesellschaft. Zwetajewa bietet ihren Lesern eine bewusst zugespitzte gleichsam wörtliche Realisierung der politischen Metaphern an, denn die Dinge im Gedicht können die Verdinglichung nur auf eine radikale Weise rückgängig machen - durch das Feuer. In dem imaginierten Brand verschwinden die Dinge der Armut ohne die Menschen zu verletzen. Hier kehrt der biblische Aspekt der Jakobsleiter zurück in den Text: «Auf der Treppe - wo die Schläfer glühend traben - / Gehen die Regenbögen - was für Farben! - / Hoch und runter.» ${ }^{17}$ Doch nicht die Engel (wie im Jakobstraum), sondern die Farben auf dem Treppenhaus, das den Brand überstanden hat, gehen die Treppen «hoch und runter». Am Ende des Gedichts entsteht statt des metaphysischen Raums an dieser Stelle im Text ein utopischer Raum der Dichtung, der es dem «lyrischen Ich» ermöglicht, jenseits der alltäglichen Armut zu existieren. Die bedrohend bleibende Armut wird aber bis zum Ende von Zwetajewas Leben für sie eine dauerhafte existenzielle Frage bleiben.

\section{Zwischen Moskau und Paris}

In den zwanziger Jahren wurde Paris $\mathrm{zu}$ einem der wichtigsten Zentren der russischen Literatur. Hier lebten die Schriftsteller Ivan Bunin, Alexander Kuprin und die Philosophen Lev Schestow, Nikolai Berdjajew und andere, für westliche Leser heute weniger bekannte russische Exilanten. ${ }^{18}$ Wie der amerikanische Literaturwissenschaftler Simon Karlinsky feststellte, lebten die meisten dieser Autoren in heute kaum vorstellbaren prekären Verhältnissen. Ein besonders tragisches Beispiel für diese Armut, so betont auch Karlinsky, war die Lage von Marina Zwetajewa, die nie gut war, sich ab 1927 aber noch einmal verschlechtern sollte. ${ }^{19}$ Diese Not war auch mit ihrer Position in der russischen EmigrationsLiteratur verbunden. Im Jahr 1928 veröffentlichte Zwetajewa in der Zeitschrift Eurasia eine kurze poetische Begrüßung des Dichters Vladimir Majakowskij, der als führende Stimme in der Sowjetunion galt und der damals Paris besuchte. Nach einer Lesung von Majakowskij in Paris schrieb Zwetajewa mit Blick auf die

17 Ebda., S. 102.

18 Vgl. hierzu Robert Harold Johnston: Paris: Die Hauptstadt der russischen Diaspora. In: Karl Schlögel (Hg.): Der große Exodus. Die russische Emigration und ihre Zentren 1917 bis 1941. München: Beck 1994, S. 260-278.

19 Simon Karlinsky: Marina Tsvetaeva: The Woman, Her World, and Her Poetry. Cambridge: CUP 1985, S. 73. 
Situation in Sowjetrussland: «Die Kraft liegt dort.» ${ }^{20}$ Darauf folgte eine heftige und ablehnende Reaktion der russischen Immigranten, die Zwetajewas Text als vermeintlichen Ausdruck ihrer Unterstützung des sowjetischen Regimes wahrnahmen. In der Folge wurden weitere Publikation von Zwetajewas Werken in den russischen Emigranten-Zeitschriften fast unmöglich. Nicht zuletzt spielte auch die politische Tätigkeit ihres Mannes Sergej Efron und dessen Annäherung an den politischen Kurs der Sowjetunion eine wesentliche Rolle für die Distanzierung der russischen Emigrationskreise von Zwetajewa. ${ }^{21}$ Zugleich blieb die Dichterin, wie bereits erwähnt, auch dem französischen literarischen Milieu fremd, obwohl sie die französische Sprache auf einem so hohen Niveau beherrschte, dass sie ein Prosawerk auf Französisch verfassen und ihr eigenes langes Gedicht ins Französische übersetzen konnte. Dennoch blieb sie für die französische Literaturszene ein unbeschriebenes Blatt. So begann für sie, in Worten ihres Übersetzers Ralph Dutli, «das Exil im Exil». 22

Im gleichen Jahr, in dem ihre Begrüßung Majakowskijs in Eurasia erschien, publizierte Zwetajewa einen Essay über die Malerin Natalia Gontscharowa. Dieser Text fungierte als poetischer Wendepunkt im doppelten Sinn: einerseits als Meilenstein für die experimentelle Zuwendung zur für die Dichterin neuen Gattung der Prosa, und andererseits als Markierung des ernüchternden Endes derjenigen Phase der Emigration, in der Zwetajewa noch nicht völlig isoliert schreiben musste. ${ }^{23}$ Doch diese zweieinhalb Jahre vor dem Treffen mit Gontscharowa erwiesen sich für Zwetajewa als höchst produktiv, und dies trotz der prekären finanziellen Lage. Während in ihrem Werk vor der Emigration nach Paris das Autobiographische fast ausschließlich in Liebesdiskursen transformiert wurde, erweiterte sich ab 1926 dessen Spielraum zunehmend. In ihren neuen Gedichten, die in Frankreich entstehen, beginnt sich Zwetajewa sowohl mit materiellen als auch mit sozialen Aspekten ihrer Existenz auf eine radikale philosophische Weise auseinanderzusetzten.

20 Marina Zwetajewa: Eurasia, 24.11.1928, S. 8.

$21 \mathrm{Zu}$ Efrons politischer Tätigkeit in Paris siehe: Mark Bassin/Glebov Sergey u. a.: Between Europe and Asia: The Origins, Theories and Legacies of Russian Eurasianis. Pittsburg PA: PUP 2015, S. 8 10.

22 Ralph Dutli: Nachwort. In: Marina Zwetajewa: Mein weiblicher Bruder: Brief an die Amazone. Übersetzung aus dem Russischen von Ralph Dutli. Berlin: Matthes \& Seitz 1995, S. 77-95, hier S. 83.

23 Auch in dem ausführlichen und wichtigen Aufsatz von Marie-Luise Bott ist von Zwetajewas erstem Prosawerk keine Rede, es ist bisher der Aufmerksamkeit der Forschung fast völlig entgangen, vgl.: Marie-Louise Botte: Marina Zwetajewa in der Emigration 1922-1939, S. 93-115. 
Zur Zeit des Treffens der beiden Künstlerinnen, das von Zwetajewas Freund, dem russisch-jüdischen Literaturkritiker Mark Slonim initiiert worden war, lebte Gontscharowa bereits dreizehn Jahre in Paris. Wie war es zu ihrer Emigration nach Paris gekommen? Nach dem großen Erfolg, den sie mit ihrer Ausstellung von siebenhundertfünfzig Gemälden in Moskau im Jahr 1913 erzielt hatte, lud Sergei Daghilev sie noch im selben Jahr nach Paris ein, um sie für die Gestaltung der Dekoration des Opern-Balletts Le Coq d'Or von Nikolai Andrejewitsch RimskyKorsakow zu gewinnen. Im Rückblick erweist sich diese Einladung als Beginn eines langen und fruchtbaren Aufenthalts in Paris, wo sie von 1915 bis zu ihrem Tode im Jahr 1962 mit ihrem Mann, dem Künstler Michail Larionow, lebte. Für Gontscharowa bedeutete Paris also der Beginn ihrer internationalen Anerkennung als russische Avantgarde-Künstlerin.

Zwetajewa kam zehn Jahre später nach Paris. ${ }^{24}$ Im Spannungsfeld einer Stadt, die zum Exilort für die eine und zum Zufluchtsort für die andere geworden war, entstand das Prosawerk der Dichterin, das zugleich ihr einziges über Paris bleiben sollte. Der Text Natalia Gontscharowa: Ihr Leben und ihr Werk, der mehr als hundert Seiten umfasst, dokumentiert diese Begegnung, die in Gontscharowas Atelier in der Rue Visconti stattgefunden hat. Er war Zwetajewas erstes Prosawerk, eine Art experimentelle Studie, die sie in einem Brief als «Nicht-Aufsatz» (ne statja) bezeichnet hat: «Ich schreibe einen großen Nicht-Aufsatz über N. Gontscharowa, die beste russische Malerin und vielleicht auch der beste russische Maler.» 25

Im Folgenden soll auf einen wichtigen Aspekt dieses vielschichtigen literarischen Portraits der Künstlerin näher eingegangen werden, der bisher kaum erforscht wurde, nämlich Zwetajewas Auseinandersetzung mit der kulturellen Bedeutung von Immigration. Um diese Dimension in Zwetajewas Prosawerk deutlich zu machen, wird zunächst Zwetajewas Darstellung der Topographie der Rue Visconti untersucht. Ihre Darstellung ermöglichte es ihr nämlich, auch den anderen Ort, der beiden Künstlerinnen vertraut war, die Moskauer Gasse ihrer Kindheit in Erinnerung zu rufen. Dabei birgt diese doppelte oder auch gespiegelte Topographie russischer Erinnerungen mit französischer Gegenwart, so mein Argument, Zwetajewas erste Überlegungen zur Bedeutung der Immigration und ihrer Wirkung auf die künstlerische Arbeit. Die Frage nach der Möglichkeit der Übersetzbarkeit von Kultur zieht sich als ein roter Faden durch Zwetajewas Schriften, die im französischen Exil entstanden sind. Es scheint mir also alles

24 Ein Jahr später war Zwetajewa gezwungen, nach Bellevue - Pariser Vorort, umzuziehen - in Paris zu wohnen kam für sie aus finanziellen Gründen nicht mehr in Frage.

25 Marina Zwetajewa: Sobranije sochinenij v semi tomah [Gesammelte Schriften in sieben Bänden]. Bd. VI. Moskau: Ellis Lak 1994, S. 376. 
andere als Zufall, dass sich Zwetajewa ausgerechnet in ihrem Essay über Gontscharowa zum ersten Mal zur Frage der Immigration geäußert hat, da ja die Frage der Übersetzbarkeit der Kunst, sowohl der bildenden als auch der literarischen, in diesem Text eine wesentliche Rolle spielt.

In einem umfangreichen Aufsatz untersucht die amerikanische Literaturwissenschaftlerin Molly Blasing die Transformationen der bildenden Kunst in Zwetajewas Werken in eine «linguistische oder poetische Form», die es ihr ermöglichte, die Frage des Selbst und des Anderen zu reflektieren. Ihr Hauptargument lautet wie folgt:

In Zwetajewas schöpferischer Welt bietet einem das Auge ein Portal in eine andere Welt an, ein Medium, das den Zugang zu einer Version des Selbst oder zu einer anderen poetischen Seele ermöglicht. Die Dichterin sucht eine Verbindung mit einer Person oder mit einem Ort, die sie in der physischen Welt nicht greifen kann. ${ }^{26}$

$\mathrm{Zu}$ Recht weist Blasing auf diesen wichtigen und bislang so gut wie unerforschten Aspekt in Zwetajewas Schreiben hin. Mit Bezug auf den hier fokussierten Essay über Natalia Gontscharowa gibt sie der Frage nach dem biographischen Zusammenhang zwischen den beiden Künstlerinnen allerdings nur sehr wenig Raum. Es lässt sich jedoch nicht genug betonen, dass sich in diesem Text eine zugleich physische, intellektuelle und poetische Begegnung niedergeschlagen hat, vor deren Hintergrund Zwetajewa die kulturelle und existenzielle Bedeutung von Immigration zum ersten Mal reflektiert.

«Keine Gasse, eine Schlucht eher», so beginnt Zwetajewa ihren Essay, und sie fährt fort:

Auf Armlänge die Wand: die Flanke des Berges. Keine Häuser, Berge, alte, uralte Berge. (Junge Berge gibt es nicht, ist er jung - ist er kein Berg, ein Berg - der ist eben alt.) Berge und Höhlen. Berg und Höhle sind ihr Heim. Keine Gasse. Schlucht eher, besser noch - ein enger Paß. So sehr keine Straße, daß ich jedes Mal, in Vergessenheit und Erwartung der Straße - dabei gibt es einen Namen, und es gibt eine Nummer! - an ihr vorüberlaufe und dessen erst direkt an der Seine gewahr werde. Also-zurück-suchen, aber die Gasse weicht aus- das ausweichende Wesen von Schluchten! Fragt doch das Bergvolk - umherhetzen, umhertappen - ist sie das? Nein, ein Haus, das sich auf einmal mit einem Hof dichtmacht so groß wie ein Platz fast, nein ein Torbogen aus dem Jahrhunderte hervorwehen, nein, bloß - eine Straße mit Schaufenstern, mit Fahrzeugen. Die ich suche, gibt es nicht. Ist verschwunden. Der Berg hat sich geschlossen und dabei Goncharowa und ihre Schätze verschluckt. Zu Goncharowa komme ich heute nicht mehr hin, und ich selber komme um. Rechte Seite, linke Seite? Platz St. Germain, die Seine? Wo-was? Und in Bezug auf welches

26 Molly Thomasy Blasing: Marina Cvetaeva and the Visual Arts. In: Sibelian Forrester (Hg.): $A$ Companion to Marina Cvetaeva. Approaches to a Major Russian Poet. Leiden/Boston: Brill 2016, S. 91-238, hier S. 194. 
Was ist dies Wo? Und plötzlich - ein Wunder! - das gibt's doch nicht! Gibt es doch, denn es ist ja da! Wirklich - sie? Natürlich sie - er der enge Paß - die Schlucht! Gleich hier, zwischen zwei Häusern, als sei nichts gewesen, als sei sie schon immer dagewesen. ${ }^{27}$

Zwetajewa eröffnet ihren Text mit der Darstellung einer topografischen Desorientierung, ein avantgardistisches Verfahren, das ihr ermöglicht auch den Lesern zu verwirren. Denn ohne die einzelnen Erwähnungen der topographischen Merkmale der Stadt könnte man nicht ahnen, dass es um Paris geht. Diese Verschiebung oder sogar Vortäuschung des konkreten Orts verweist darauf, dass die Darstellung der Stadt Paris nicht im Zentrum des Essays stehen wird, sondern dass es im Essay um das Gegenteil geht, nämlich um die Möglichkeit, über die physischen Orte $\mathrm{zu}$ schreiben, die man - um noch einmal mit den Worten Blasings zu sprechen - nicht «greifen kann». Im gesamten Text wird die Rue Visconti also nicht mit ihrem Namen genannt. Nicht als eine Straße, sondern als ein Teil der Natur, oder als eine Schlucht aus Tausendundeiner Nacht inszeniert Marina Zwetajewa den Aufenthaltsort von Natalja Gontscharowa. In dieser Darstellung des Ateliers der Künstlerin spielt das urbane Element kaum eine Rolle - zweifellos nicht zufällig, da eine von Zwetajewas Hauptthesen über Gontcharowas Kunst genau darin besteht, dass der Ursprung ihrer avantgardistischen Kunst nicht in der Metropole liegt, sondern in der Begegnung mit dem russischen Dorf, in dem sie aufwuchs und in der Natur, die sie seinerzeit umgeben hatte. Doch es war keine Begegnung im folkloristischen Sinne, sondern ein Prisma der Wahrnehmung, oder in Zwetajewas Worten: «Das Ländliche nicht als Klasse, sondern als Lebensform». ${ }^{28}$ Sogar in Moskau habe Gontscharowa immer wieder das Ländliche gemalt. $^{29}$

Am Ende des ersten Kapitels macht Zwetajewa eine linguistische Bemerkung zu dem Wort «Aufenthaltsort», die von der philosophischen Bedeutung nicht zu trennen ist. Hier heißt es:

Der Ort, wo ein Ding immer ist, der ist auch Aufenthaltsort - was für ein wunderbares Wort übrigens, es vermittelt in einem sowohl das Gegenwärtig-Sein als auch das Andauern, die Lage im Raum und das Erstrecken in der Zeit, was für ein langgestrecktes, was für ein geräumiges Wort. So ist Rußland zum Beispiel der Aufenthaltsort von Wehmut, über die ebenso befremdlich wie über den Wind zu sagen wäre: sie wohnt. Doch - sie wohnt! ${ }^{30}$

27 Marina Zwetajewa: Gedichte, Prosa. Übersetzung von Fritz Mierau. Leipzig: Reclam 1987, S. 133.

28 Marina Zwetajewa: Gedichte, Prosa, S. 243.

29 Ebda.

30 Ebda., S. 135. 
Der Aufenthaltsort enthält also nicht nur die räumliche Komponente, sondern auch die zeitliche. Hierin kann man auch Zwetajewas poetische Definition der Immigration erkennen. Nicht zufällig gibt die Dichterin ausgerechnet Russland als Aufenthaltsort von Wehmut an, wird doch im Chronotopos des Pariser Hauses von Gontscharowa das vorrevolutionäre Moskau erinnert.

Durch die während einer der zahlreichen Besuche im Atelier zufällig wahrgenommene Aufschrift einer Holzkiste - «Trjehprudnuj» (Dreiteich) - entdeckt Zwetajewa, dass sie beide in Moskau in derselben Gasse groß geworden sind, und zwar in benachbarten Häusern. Im gemeinsamen Hof stand eine weiße Pappel, die für Zwetajewa zum Symbol ihrer Kindheit wurde. Zu der Zeit, als sie den Essay schrieb, war das alte Haus der Zwetajew-Familie längst zerstört. Sie hatte das Haus noch vor der Revolution, nach dem frühen Tod der Mutter, verlassen müssen, zerstört wurde es dann einige Zeit nach den Ereignissen vom Oktober 1917. Der Baum ihrer Kindheit aber, die weiße Pappel, überstand die Zeiten. Mit dem Haus verband Zwetajewa nicht nur ihre Kindheit, sondern eine ganze Welt, etwa die verschwundene Welt der russischen Intelligenzija, der ihre beiden Eltern angehört hatten. Das Haus war der «Aufenthaltsort» (im obigen Sinne) der Bücher ihres Vaters, der Kunstgeschichtsprofessor war, und der Musik ihrer Mutter, einer begabten Pianistin, die beide die besondere Atmosphäre ihrer Kindheit prägten. Im Jahr der Revolution sah Zwetajewa das Haus zum vorletzten Mal, wenn auch nur von außen:

Ich schließe die Augen - da steht es. Ich öffne sie - es ist weg. Die Pappel hatte man nicht abgeholzt. Später vielleicht. In der Dreiteichgasse war ich nicht mehr. Werde ich auch nicht mehr sein, auch dann nicht, wenn die Druckerei Lewenson - schräg gegenüber von uns ehemals -, wo ich mein erstes Buch drucken ließ, irgendwann einmal mein letztes drucken werde. ${ }^{31}$

Diesem Versprechen ist Zwetajewa treu geblieben. Es war dies eine anti-nostalgische Erinnerungsgeste, man könnte auch sagen eine Umkehrung der Geste von Lots Frau, da Zwetajewa bewusst war, dass der Rückblick auf den zerstörten Ort die Vergangenheit nicht wieder lebendig machen kann.

Für Zwetajewa wurde Gontscharowa, die für sich selbst bezeugt hat, sie habe immer nach Osten gewollt, sei aber gen Westen gezogen, zu einer Vermittlerin der russischen Kunst in Frankreich und in Europa insgesamt. In ihrer Periodisierung von Gontscharowas Werken schlägt Zwetajewa die folgende Formel vor: Eine Phase «Russland» und eine Phase «Nach-Russland», auf keinen Fall aber «Russland» und «Emigration». Sie verstand Gontscharowas künstlerischen Werdegang

31 Ebda., S. 157. 
als einen organischen Übergang und nicht als einen Umbruch, da die Werke der Malerin, ebenso wie diese selbst, sich in Frankreich gleichsam einlebten. Dass dies für die Dichterin alles andere als selbstverständlich ist, wird an anderer Stelle deutlich, wo Zwetajewa Einwanderung grundsätzlich als einen Bruch im Erstrecken der Zeit wahrnimmt - «das lebend als aus dem Kreis der Lebenden Ausgeschlossensein.» ${ }^{32}$ Zugleich verbirgt diese existenzielle Formulierung eine Zeugenschaft davon, dass ihre eigene Erfahrung genau das Gegenbild zu derjenigen war, die Gontscharowa machte. Anders als für die Künstlerin bedeutete die Umsiedlung nach Frankreich für Zwetajewa wirklich einen Zeitbruch, da ihre Dichtung in der neuen Heimat nicht übersetzt wurde, vielleicht unübersetzbar blieb, sowohl für die französische Leserschaft als auch für den russischen Emigrantenkreis. Umso wichtiger war es für Zwetajewa, die Moskauer Gasse in ihrem Pariser Essay als einen gemeinsamen Erinnerungsort zu konstruieren, und zwar nicht nur aus nostalgischen, sondern vielmehr aus poetologischen Gründen, um nämlich die Gemeinsamkeit auch in der Kunst mittels eines avantgardistischen Schreibens zu offenbaren oder nachzudichten.

Nachträglich könnte man sagen, dass die Vollendung des Essays im März 1929 in Meudon für Marina Zwetajewa das Ende ihres Schreibens über Paris markierte. Es war dabei kein einseitiger Versuch von Marina Zwetajewa, die andere Kunst und die Kunst der anderen zu verstehen. Es gibt einen überraschenden Hinweis darauf, dass Natalja Gontscharowa diese doppelte Gemeinsamkeit ebenso empfunden hat. In einem Nebensatz machte Zwetajewa eine Anmerkung über Gontscharowas poetische Kraft des Sehens in allen Kunstbereichen, die ihr unter anderem ermöglichte, Zwetajewas metaphorische Sprache $\mathrm{zu}$ verstehen: «[...] und Gontscharowa, die nie Verse schrieb, die nie in Versen gelebt hat, begreift, weil sie sah und erkannte.» ${ }^{33}$ Allerdings wusste Zwetajewa seinerzeit nicht, dass Gontscharowa tatsächlich einmal versucht hat, Gedichte zu schreiben. Erst vor wenigen Jahren wurde bekannt, dass die Malerin Zwetajewa ein Gedicht gewidmet hat, in dem sie sich und die Freundin «Schwestern» ohne gemeinsame Eltern nennt; leider aber hat sie dieses der Dichterin nie gezeigt:

\footnotetext{
Конечно мы сестры с тобой,

Но не по отцу, не по матери,

А по тополю белому, по тени, что падала

Утром и вечером,

На твой двор и мой,

По ветру залетному,
}

32 Ebda., S. 241.

33 Ebda., S. 231. 
Что листьями сыпал, Осенними желтыми, На мой двор и твой, По блеску желтому, В серых и карих глазах. По ритму четкому, В мазках и словах. ${ }^{34}$

Selten ist in der Kunstgeschichte eine solche Gabe zu finden. Die Wahlverwandtschaft zwischen diesen beiden großen Künstlerinnen, die Gontscharowa in diesem Gedicht zum Ausdruck bringt und reflektiert, oder genauer gesagt mit Worten etabliert, gründete in mehreren Lebensbereichen. Gleich am Anfang evoziert die Malerin den Raum der Jugend, der durch die fast magische weiße Pappel und durch den gemeinsamen Hof, der im Gedicht zweimal erwähnt wird, dargestellt wird. Erst nachdem Gontscharowa die topographische Symmetrie zwischen sich und der Freundin hergestellt hat, nennt sie den wichtigsten Grund ihrer nichtbiologischen Verwandtschaft. In ihrer klaren Lyrik offenbart sie den wichtigsten Aspekt der gegenseitigen Verbundenheit - die Präzision der künstlerischen Sprache. So entsteht zwischen beiden Künstlerinnen ein gemeinsamer «Aufenthaltsort» der künstlerischen Arbeit, der in ihren literarischen Texten aufscheint, die auf Russisch in Paris verfasst wurden.

\section{Euer Paris}

Anfang der dreißiger Jahre reflektierte Zwetajewa sowohl in ihren Tagebüchern als auch in Briefen an Freundinnen ihre sich verschlechternde Lage und ihre intellektuelle Isolation. In einem Eintrag in ihren Arbeitsheften findet man die folgende Notiz über sich selbst: «Mein Misserfolg in der Emigration liegt darin, dass ich Nicht-Emigrantin bin, dass ich dem Geist nach, das heißt der Luft und der Spannweite nach, dort bin, dorthin, von dorther spreche.» ${ }^{35}$ Diese auf keine Weise beschönigende Überlegung gibt eine poetische Definition des Exils als das Schreiben und das Sprechen von «dort». In diesem Sinne bedeutet für Zwetajewa

34 «Of course, you and I are sisters / But not by father, nor mother / But by the white poplar / And the shadows that fell / Morning and evening / On your yard and mine. / By the visiting wind / That sprinkled yellow / Autumn leaves / On your yard and mine / By that yellow spark / In our grey and brown eyes. / By the precise rhythm in our brushstrokes and words.» Übersetzung von Molly Thomasy Blasing: Marina Cvetaeva and the Visual Arts, S. 218.

35 Zwetajewa zitiert nach Walentina Korosteljowa: XX Vek: Poety Russkije [Das zwanzigste Jahrhundert: Russische Dichter], Moskau: DirectMedia 2014, S. 89. Meine Übersetzung. 
die Immigration die Möglichkeit von «hier» zu schreiben. Das würde «Ankommen» bedeuten. Ein kurzes Gedicht mit dem Titel «Holzspan» aus dem gleichen Jahr 1931, in dem sie noch ein einziges Mal über Paris schreiben wird, bestätigt dies:

Der Eifelturm in Reichweite! Reich es und klettere auf/ Aber jeder von uns/ hat solches gesehen, ich sage Euch, und sieht auch heutzutage./ Euer Paris scheint für uns langweilig und hässlich zu sein./ Russland, mein Russland, warum brennst Du so hell ${ }^{36}$

Diese Strophen scheinen die kulturelle und zugleich poetologische Einsicht - Exil bedeutet Sprechen von «dort», Immigration bedeutet Sprechen von «hier» - noch in zugespitzter Form zum Ausdruck zu bringen. Der Grund für ihre existenzielle und poetologische Selbst-Positionierung als eine Nicht-Immigrantin liegt also nicht in der Unfähigkeit, das Hier und Jetzt darzustellen. Vielmehr geht es darum, dass Russland für Zwetajewa über viel größere sowohl ästhetische als auch kulturelle Anziehungskraft verfügt als Paris, trotz ihrer scharfen Ablehnung der sowjetischen Politik.

Es gab aber noch einen anderen, vielleicht weniger evidenten Grund dafür, warum ausgerechnet Marina Zwetajewa Frankreich als Ort des «Nicht-Ankommens» wahrgenommen hat. Es scheint so zu sein, dass Zwetajewas Einstellung zu Frankreich und zur französischen Kultur von Anfang an durch eine gewisse Ambivalenz geprägt war. Diese Ambivalenz erreichte ihren Höhepunkt 1932, nachdem Zwetajewa daran gescheitert war, sich im Pariser literarischen Milieu bekannt zu machen und von den französischen Schriftstellern wahrgenommen zu werden. Mit der Übersetzung ihres langen Gedichts «Le Gars...», für das Natalija Gontscharowa die Illustrationen beisteuern sollte, mache sich die Dichterin Hoffnungen, die französische literarische Szene auf sich aufmerksam machen zu können. Dank der Bemühungen ihrer Freundin Elena Iswolskaja gelang es Zwetajewa, zu einer Lesung in einem literarischen Salon eingeladen zu werden. Leider fehlen fast alle genauen Angaben zu dieser Lesung, so dass wir heute nicht viel über sie wissen. In der Forschung herrscht Unklarheit sowohl über den Ort, an dem die Lesung stattfand, als auch über die Teilnehmer. Doch über eins sind sich die Literaturhistoriker einig, nämlich dass es ein tragisches Fiasko war. Die in sozialen Kategorien in den Augen der Franzosen «deklassierte» große Dichterin aus Russland, die mit ihrer sehr alten Kleidung und mit einer besonderen, für den russischen Modernismus typischen Art der lyrischen Deklamation das avantgardistische Gedicht vortrug, blieb für die literarische Elite von Paris höchst befremdlich und insgesamt völlig unverständlich. Noch im gleichen Jahr machte sie einen

36 Marina Zwetajewa: Sochinenija. Bd. III, S. 3. Meine Übersetzung. 
weiteren - und letzten - Versuch, mit den Pariser Schriftstellern in einen Dialog zu treten, dieses Mal galt der Versuch der berühmten Amerikanerin Natalie Barney. Bis heute ist es unbekannt, unter welchen Umständen das Treffen zwischen den beiden Schriftstellerinnen stattfand. ${ }^{37}$ Die einzige Erwähnung von diesem Ereignis ist in Zwetajewas Essay «Mon frère féminin. Lettre à L'Amazone», den sie 1932 auf Französisch verfasste. Die erste französische Ausgabe dieses Textes, der eine literarische Reaktion auf Barneys Buch Pensées d'une Amazone darstellt, erschien 1979, die erste russische Ausgabe erschien sogar erst 1987. Die Literaturwissenschaftlerin Ulrike Jekutsch fasst Zwetajewas Brief an Barney wie folgt zusammen: «Wenn Natalie Barneys Pensées d'une Amazone eine Verteidigung und Propagierung lesbischer Beziehungen unter kreativen Frauen darstellen, so versucht Cvetajewa eine partielle Widerlegung, indem, wie sie schreibt, gerade die Frage ins Zentrum rücke, die Barney nur am Rande erwähnt hat, nämlich die nach dem Kind.» ${ }^{38}$ Diese Episode in Zwetajewas literarischer Biographie scheint mir nicht nur deshalb so wichtig zu sein, weil Zwetajewa hier zum ersten Mal ihre eigene Erfahrung in Liebesbeziehungen mit Frauen in einem literarischen Text reflektiert und in eine andere Sprache übersetzt, sondern auch deshalb, weil sie dies in einem Dialog mit einer anderen translingualen Autorin versucht. Auch wenn sie Barney schreibt, dass es ihr nur darum ginge, dass die amerikanisch-französische Autorin ihr zuhöre: «Hören Sie mir zu - Sie brauchen nicht zu antworten», ${ }^{39}$ erhoffte sie natürlich eine Antwort. Die Tatsache, dass der Dialog mit Natalie Barney von Zwetajewa zu Recht als Dialog mit der zeitgenössischen französischen Literatur assoziiert wurde und dass er gescheitert ist, hat sicherlich nicht nur zu Zwetajewas Selbst-Wahrnehmung als Nicht-Immigrantin beigetragen, sondern auch ihre kulturelle Ambivalenz Frankreich gegenüber verstärkt.

Noch im selben Jahr formulierte Zwetajewa in ihrem Essay über den Dichter und Maler Maximilian Woloschin, mehr als zwanzig Jahre nach ihrem ersten Besuch in Paris:

Wir verspürten nie eine Verwandtschaft mit Frankreich. Wir sind verschieden. Wir liebten und lieben es, wir waren in Frankreich verliebt, sind es vielleicht noch immer oder werden es wieder sein, unsere Beziehung zu Frankreich ist Faszination ohne Verständnis, denn

37 Karlinsky, Simon: Marina Tsvetaeva: The Woman, Her World, and Her Poetry. Cambridge: CUP 1985, S. 208.

38 Ulrike Jekutsch: «Mein weiblicher Bruder»: Rollenkonflikte bei Marina Cvetaeva. In: Doris Ruhe (Hg.): Geschlechterdifferenz im interdisziplinären Gespräch. Würzburg: Königshausen \& Neumann 1998, S. 151-171, hier S. 154.

39 Marina Zwetajewa: Mein weiblicher Bruder: Brief an die Amazone. Übersetzung aus dem Russischen von Ralph Dutli. Berlin: Matthes \& Seitz 1995, S. 10. 
einen anderen zu verstehen bedeutet, wenigstens für eine Stunde er zu werden. Wir aber sind außerstande, auch nur für eine Stunde Franzosen zu werden. Kraft und Ursprung unserer Faszination liegen zur Gänze - in der Fremdheit. Unser Verwandter, unsere Verwandtschaft ist der bescheidene und unansehnliche Nachbar Deutschland - in den wir nie verliebt waren, auch wenn wir ihn einst in Gestalt der besten Köpfe und Herzen unseres Landes liebten. Man ist ja auch nicht in sich selbst verliebt. ${ }^{40}$

In diesem Abschnitt offenbart die Dichterin in Umschreibungen eines Liebesdiskurses nicht nur ihre Haltung zur französischen Kultur, sondern auch diejenige Deutschland gegenüber, und damit die Quintessenz ihrer Kulturphilosophie, die auf ihre eigenen Erfahrungen in der Emigration zurückgreift. Um eine andere Kultur zu verstehen, so Zwetajewa, sollte man über die ethische Gabe verfügen, sich als der Andere imaginieren zu können. Damit die beiden Kulturen füreinander übersetzbar werden können, muss dieser Prozess aber wechselseitig verlaufen. Ohne diesen poetischen und ethischen Impuls, der das fremde Element in der anderen Kultur, das über eine große Anziehungskraft verfügt, de-fetischisiert, um sie zu verstehen, kann kein interkultureller Raum entstehen. Im Sinne Wolfgang Isers könnte man deshalb an dieser Stelle auch argumentieren, dass Zwetajewa nach einer kulturellen Übersetzbarkeit strebte, die nur dann eintrat, wenn sie einen Diskurs schaffte, in dem es möglich war, den «Raum zwischen Fremdheit und Vertrautheit zu verhandeln.» ${ }^{41}$ Umso wichtiger scheint Zwetajewas Essay über Gontscharowa für das Verstehen von Zwetajewas Exilzeit in Frankreich zu sein, da er auch der einzige Pariser Text bleiben wird, in dem Paris als Ort der Begegnung zwischen Kunst und Dichtung Thema wird, als ein Raum also, der zwischen den Kulturen existieren kann.

\section{Literaturverzeichnis}

Asadowski, Konstantin: Orpheus und Psyche. Vorwort. In: Rainer Maria Rilke und Marina Zwetajewa: Ein Gespräch in Briefen. Frankfurt a. M.: Suhrkamp 1998, S. 7-45.

Blasing, Molly Thomasy: Marina Cvetaeva and the Visual Arts. In: Sibelian Forrester (Hg.): $A$ Companion to Marina Cvetaeva Approaches to a Major Russian Poet. Leiden/Bosten: Brill 2016, S. 191-238.

Bassin Mark/Glebov Sergey u. a.: Between Europe and Asia: The Origins, Theories and Legacies of Russian Eurasianis. Pittsburg PA: Pittsburg University Press 2015.

40 Marina Zwetajewa: Begegnungen mit Maximilian Woloschin, Andrej Belyj und Rudolf Steiner. Übersetzung von Ilma Rakusa und Rolf-Dietrich Kiel. Dornach: Verlag die Pforte 2000, S. 93.

41 Wolfgang Iser: On Translatability. In: Surfaces IV, 307 (1994), S. 1-13, hier S. 9. 
Botte, Marie-Louise: Marina Zwetajewa in der Emigration 1922-1939. In: Simon-Pierre Hamelin, 101 rue Condorcet, Clamar. Übersetzung von Regina Keil-Sagawe. Hamburg: Osburg Verlag 2017, S. 93-115.

Dutli, Ralph: Nachwort. In: Marina Zwetajewa: Mein weiblicher Bruder: Briefan die Amazone. Übersetzung von Ralph Dutli. Berlin: Matthes \& Seitz 1995, S. 77-95.

Iser, Wolfgang: On Translatability. In: Surfaces IV, 307 (1994), S. 1-13.

Jekutsch, Ulrike: «Mein weiblicher Bruder»: Rollenkonflikte bei Marina Cvetaeva. In: Doris Ruhe (Hg.): Geschlechterdifferenz im interdisziplinären Gespräch. Würzburg: Königshausen \& Neuman 1998, S. 151-171.

Johnston, Robert Harold: Paris: Der Hauptstadt der russischen Diaspora. In: Karl Schlögel (Hg.): Der große Exodus. Die russische Emigration und ihre Zentren 1917 bis 1941. München: Beck 1994, S. 260-278.

Karlinsky, Simon: Marina Tsvetaeva: The Woman, Her World, and Her Poetry. Cambridge: CUP 1985.

Korosteljowa, Walentina: XX Vek: Poety Russkije [Das zwanzigste Jahrhundert: Russische Dichter]. Moskau: Direct Media 2014.

Lukács, Georg: Geschichte und Klassenbewusstsein. Studien über marxistische Dialektik. Berlin: Malik-Verlag 1923.

Stock, Ute: The Ethics of the Poet: Marina Tvetaeva's Art in the Light of Conscience. Leeds: Maney Publishing 2005.

Zwetajewa, Marina: Gedichte, Prosa. Übersetzung von Fritz Mierau. Leipzig: Reclam 1987.

Zwetajewa, Marina: Sochinenija [Gesammelte Schriften]. Moskau: Chudozestvennaja Literatura 1990.

Zwetajewa, Marina: Gruß vom Meer. Übersetzung von Felix Phillip Ingold. München: Hanser Verlag 1994.

Zwetajewa, Marina: Sobranije sochinenij v semi tomah [Gesammelte Schriften in sieben Bändern]. Moskau: Ellis Lak 1994.

Zwetajewa, Marina: Mein weiblicher Bruder: Brief an die Amazone. Übersetzung von Ralph Dutli. Berlin: Matthes \& Seitz 1995.

Zwetajewa, Marina: Begegnungen mit Maximilian Woloschin, Andrej Belyj und RudolfSteiner. Übersetzung von Ilma Rakusa/Rolf-Dietrich Kiel. Dornach: Verlag die Pforte 2000. 